\title{
Le vent contre la vase
}

Un projet de restitution de moulin à vent dans l'arsenal de Rochefort The wind against the mud. A project for the restoration of the Rochefort arsenal windmill

\section{Pierre Gras, David Plouviez et Denis Roland}

\section{OpenEdition Journals}

Édition électronique

URL : http://journals.openedition.org/artefact/6917

DOI : 10.4000/artefact.6917

ISSN : 2606-9245

\section{Éditeur :}

Association Artefact. Techniques histoire et sciences humaines, Presses universitaires du Midi

\section{Édition imprimée}

Date de publication : 7 janvier 2021

Pagination : 363-398

ISBN : 978-2-8107-0706-5

ISSN : 2273-0753

\section{Référence électronique}

Pierre Gras, David Plouviez et Denis Roland, «Le vent contre la vase », Artefact [En ligne], 13 | 2020, mis en ligne le 23 décembre 2020, consulté le 25 décembre 2020. URL : http:// journals.openedition.org/artefact/6917 ; DOI : https://doi.org/10.4000/artefact.6917

\section{(2) $(0 \Theta \Theta$}

Artefact, Techniques, histoire et sciences humaines est mise à disposition selon les termes de la Licence Creative Commons Attribution - Pas d'Utilisation Commerciale - Pas de Modification 4.0 International. 


\title{
Le vent contre la vase
}

\author{
Un projet de restitution de moulin à vent dans l'arsenal \\ de Rochefort \\ Pierre Gras, David Plouviez et Denis Roland
}

\section{Résumé}

En septembre 2016, à Rochefort en Charente-Maritime, l'Association du Moulin de l'Arsenal de Rochefort (AMAR) a été fondée avec l'ambition de restituer un moulin de 1805 destiné à dévaser l'entrée de la double forme de radoub de l'arsenal. Conçu par l'ingénieur-constructeur de la Marine Jean-Baptiste Hubert, ce moulin fournissait l'énergie nécessaire afin d'animer un bateau racleur empêchant le dépôt de la vase. Ce projet permet de reconsidérer l'histoire des techniques et de l'innovation dans le contexte d'un arsenal de marine, du $\mathrm{xvII}^{\mathrm{e}}$ siècle à l'ère industrielle, et fournit ainsi la possibilité d'une médiation patrimoniale plus cohérente. Toutefois, cette restitution conduit à questionner sa nature et son statut dans le contexte d'une législation et de procédures administratives touchant à la navigation, au patrimoine et à l'environnement, et interroge sur sa capacité à intégrer les politiques touristiques mises en œuvre par les collectivités publiques locales.

\section{Mots-clés}

moulin à vent, arsenal, ingénieur, Jean-Baptiste Hubert, restitution, patrimoine

99 Pierre Gras, David Plouviez et Denis Roland, «Le vent contre la vase. Un projet de restitution de moulin à vent dans l'arsenal de Rochefort », Artefact, 13, 2020, p. 363-398. 


\section{The wind against the mud. A project for the restoration of the Rochefort arsenal windmill}

\section{Abstract}

In September 2016, the Rochefort Arsenal Windmill Association was founded in Rochefort, Charente-Maritime, with the aim restoring a windmill from 1805, created to remove mud from the arsenal's dry dock. Designed by the Navy structural engineer Jean-Baptiste Hubert, this windmill provided the energy required to power a scraper boat which would prevent the build-up of mud. This project allows us to reconsider what we know about techniques and innovation in the context of a naval arsenal from the $17^{\text {th }}$ century to the industrial era, thus facilitating more coherent communication of our heritage. However, this restoration has raised questions regarding its nature and status within legislation and administrative procedures with respect to sailing, heritage and the environment, and the project's ability to integrate the tourism policies implemented by local public 364 authorities.

\section{Keywords}

windmill, dockyard, engineer, Jean-Baptiste Hubert, reconstruction, heritage 


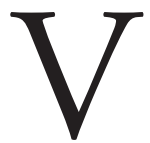

ille neuve du XvII ${ }^{\mathrm{e}}$ siècle, la naissance de Rochefort est intimement liée à la volonté de l'État d'y créer un arsenal maritime permettant la construction, l'entretien et l'armement d'une flotte de guerre permanente. Les contraintes du site, notamment liées à la nature des sols et au régime fluvial de la Charente, conjuguées à la difficile mâ̂trise d'un outil guerrier émergeant, ont représenté des défis techniques imposant la synthèse de savoirs et de savoir-faire spécifiques. L'arsenal de Rochefort acquiert dès son origine un statut particulier dans le réseau des bases navales françaises en devenant un lieu privilégié d'expertise et de connaissances grâce à une médiation permanente entre savants, architectes, ingénieurs, artisans et ouvriers ${ }^{1}$. À l'écart des grandes destructions subies par ses homologues à Brest, Lorient ou Toulon, cette infrastructure apparaît encore aujourd'hui comme un ensemble industriel complet dédié à la construction navale militaire de la période $\mathrm{XVII}^{\mathrm{e}}$-XIX ${ }^{\mathrm{e}}$ siècle, essaimant le long des méandres de la Charente un patrimoine d'une grande variété et d'une qualité exceptionnelle à l'échelle européenne.

Depuis les années 1970, ce patrimoine est l'une des bases du renouveau puis du dynamisme de la ville de Rochefort ${ }^{2}$. Mais ce rebond est en partie lié à la volonté des acteurs locaux d'éviter la muséification du site en imaginant, en inventant et en innovant avec les infrastructures existantes. De fait, cette requalification, qui s'appuie pour l'essentiel sur un patrimoine industriel et technique singulier (corderie, bassin de radoub, fosses aux mâts, etc.), passe par une historicisation permanente afin de montrer la logique de fonctionnement et la cohérence d'un arsenal de rivière, d'autant que celui-ci s'inscrit sur un espace très étendu³. Pourtant, l'ancienneté de ce patrimoine, pour l'essentiel des $\mathrm{XVII}^{\mathrm{e}}$ et XVIII ${ }^{\mathrm{e}}$ siècles, et la construction, à partir de la fin des années 1990, d'une réplique de l'Hermione, un navire

\footnotetext{
1. Nous reprenons ici l'idée formulée par Simon Schaffer pour évoquer la place des arsenaux de marine dans la construction des connaissances à l'époque moderne et au XIX siècle : Schaffer, 2007, p. 283.

2. Cet élan local est à remettre dans un contexte plus global d'intérêt pour le patrimoine scientifique, industriel et technique. Les travaux de Marina Gasnier permettent une remise en perspective de cette dynamique (Gasnier, 2011, 2018).

3. En dehors de l'arsenal proprement dit, il faut considérer l'ensemble des infrastructures en dehors de son périmètre telles les fosses aux mâts de la Gardette, le bâtiment pyrotechnique du Vergeroux ou encore la fontaine du fort Lupin destinée à remplir les tonneaux d'eau avant un appareillage du navire. Entre l'arsenal et la rade d'Aix, les éléments de l'arsenal s'étendent sur une vingtaine de kilomètres en bordure de la Charente.
} 
de 1779 , ont placé le curseur de la médiation auprès du public presque exclusivement sur l'Ancien Régime. L'absence d'éléments patrimoniaux relevant de l'ère industrielle ${ }^{4}$, une période qui représente pourtant un âge d'or pour les arsenaux européens ${ }^{5}$, ne permet pas à cette médiation d'inclure le XIx $x^{e}$ siècle à son discours. C'est dans ce contexte qu'est né en 2016 le projet de restitution d'un moulin à vent destiné à draguer les vases, conçu par l'ingénieur-constructeur Jean-Baptiste Hubert en 1805. Un moulin à vent pour symboliser l'entrée de l'arsenal dans « la révolution industrielle » n'a de prime abord rien d'évident d'un point de vue historique, autant que le principe d'une restitution qui pose de nombreuses questions au regard des habitus et du corpus législatif relatif à la préservation et à la restauration du patrimoine. Néanmoins, l'objectif est de montrer que ce moulin représente non seulement l'outil idoine pour remettre en perspective une histoire des techniques portuaires à l'échelle locale et globale, mais qu'il permet aussi corrélativement de réinscrire le patrimoine de l'arsenal dans un temps long de la recherche de l'innovation et ainsi proposer une grille de lecture plus complète aux visiteurs du site.

\section{Rochefort, le fleuve et l'ingénieur Jean-Baptiste Hubert}

\section{Atouts et contraintes d'un site fluvial}

Le projet de restitution de ce moulin à vent a une forte signification historique et patrimoniale tant il rappelle la cohabitation difficile entre l'arsenal et la Charente. Pourtant, lorsque le site de Rochefort est choisi pour abriter les navires de guerre en 1666, son éloignement de la mer $-25 \mathrm{~km}$ - au bord d'une rivière étroite et vaseuse est perçu dans un premier temps comme un atout. La plupart des ports de l'époque sont situés aux abords de fleuves ou de rivières, et la Charente offre une profondeur acceptable pour le tirant d'eau des navires qui ont la possibilité de relâcher sans s'exposer à l'ennemi ${ }^{6}$. L'échouage lié aux marées n'est pas redouté car les coques peuvent toucher sans dommage, précisément grâce aux vases de

4. La forme de radoub dite "forme Napoléon III », construite entre 1853 et 1861, est l'élément du XIX $^{\mathrm{e}}$ siècle le plus remarquable dans l'arsenal d'aujourd'hui.

5. Acerra, 2009.

6. Acerra, 1993, p. 27-43. 
fond, et les ressources naturelles de l'arrière-pays plaident à l'évidence pour Rochefort $^{7}$, faute d'un autre site cumulant autant d'avantages et libre sur la façade atlantique. La gestion du fleuve devient vraiment problématique à partir du moment où la monarchie décide de faire de Rochefort un arsenal complet destiné à construire, entretenir et armer la flotte à partir de 1669. La construction de la corderie de mars 1666 à juin 1669 avait donné un avant-goût du défi auquel architectes et ingénieurs allaient être confrontés en permanence à Rochefort ${ }^{8}$. Mais cette infrastructure en rejoint beaucoup d'autres dans le royaume au XVII ${ }^{\mathrm{e}}$ siècle où la maîtrise de l'eau devient un enjeu technique tout autant que politique?

L'envasement du fleuve est ensuite une préoccupation permanente durant toute l'activité de l'arsenal, particulièrement à l'entrée de la double forme de radoub, une infrastructure stratégique pour la construction et l'entretien des navires (Fig. I, cahier couleur). Celle-ci avait été réalisée à partir de 1683 de manière oblique par rapport au fleuve afin d'éviter le report des vases devant ses portes, une disposition qui n'eut aucun effet. Fréquemment, l'accès à la double forme est obstrué et contraint à un nouveau dragage, comme c'est le cas en 1787 :

Ce travail est d'autant plus long que les portes ont resté longtemps fermées, les vases accumulées y acquièrent un volume et une consistance qui augmentent les difficultés et par conséquent la dépense, et il est arrivé que cette opération [...] n'était pas achevée, il a fallu que quelques fois remettre à quinze jours l'ouverture des portes, retardement qui peut être très préjudiciable ${ }^{10}$.

Le limon charrié par la Charente se dépose et s'infiltre partout obligeant à des travaux d'entretien permanents qui s'élèvent, selon l'estimation de Martine Acerra, à près de 1,5 million de livres tournois de la fondation de l'arsenal à $1775^{11}$. Ces opérations coûteuses contraignent également à

7. Larrière-pays de Rochefort est riche en bois de construction, en chanvre, en fer et en vivres. Mémain, 1937 ; Plouviez, 2014.

8. La corderie est le premier bâtiment à être édifié au bord de la Charente sur une berge meuble qui impose à son concepteur, François Blondel, de créer des fondations sur un radeau de madriers en chêne, une technique empruntée aux travaux hydrauliques hollandais : Blondel, 1675, p. 656-659. 9. Morera, 2005, p. 49-60 ; on se reportera également à la thèse de l'auteur (Morera, 2011).

10. Archives nationales [désormais AN], Marine, $\mathrm{D}^{2} 34, \mathrm{f}^{\circ}$ 562, Bonnamy de Bellefontaine à Castries, 15 mars 1787.

11. Acerra, 1985, 1998, p. 66. 
chaque fois à une logistique complexe puisque le curage est réalisé tout au long de l'Ancien Régime par une drague à augets reposant sur un mécanisme de 14 charrues mues par 56 bœufs $^{12}$.

\section{L'innovation technique dans les arsenaux de marine}

L'innovation de Jean-Baptiste Hubert pour contourner en partie ces difficultés n'est réalisée qu'au cours de l'Empire, mais il est nécessaire de replacer son activité dans une perspective plus globale. Pour aussi singulière qu'ait été la figure de cet ingénieur, sa trajectoire s’inscrit dans une période, de la fin de la guerre de Sept Ans à la Restauration, qui correspond à un long moment de restructuration et de rationalisation des arsenaux français. Si le manque d'entretien des infrastructures au cours de la première moitié du XVIII ${ }^{\mathrm{e}}$ siècle contraint la Marine à les réparer ensuite, ces lourds travaux sont sous-tendus par une réflexion sur la nécessité d'une redistribution spatiale des différentes aires de l'arsenal ainsi que la mise en ouvre d'outils destinés à améliorer les phases productives de ses chantiers et de ses ateliers. Au milieu du XVIII ${ }^{\mathrm{e}}$ siècle, l'intendant Le Norman de Mési pose les jalons d'une rationalisation de l'espace de Rochefort en tentant de lui donner " un ordre exact ${ }^{13}$ ». À Brest, c'est également à partir de cette époque que l'ingénieur Choquet de Lindu révise l'agencement complexe des rives de la Penfeld en faisant construire des quais, des magasins le long des formes de construction, des fontaines, tout en généralisant l'emploi des moulins à scier les bois. Pour paraphraser l'ingénieur-constructeur Pierre-Alexandre Forfait, il y a sans doute encore beaucoup de travail pour faire de Brest un arsenal commode après les travaux dirigés par Choquet de Lindu ${ }^{14}$, mais ils permettent d'accroître sa productivité. Du côté du Levant, Toulon se dote de sa première forme de radoub d'après les plans de l'ingénieur Antoine Grognard entre 1774 et $1778^{15}$, la première infrastructure de ce type en Méditerranée. Enfin, l'énergie vapeur est utilisée précocement par

12. Les " augets" désignent les contenants en lien avec les charrues qui raclaient le lit du fleuve à mesure qu'avançaient les bœufs sur la berge.

13. Bibliothèque nationale de France, nouvelles acquisitions françaises, 126, Mémoire sur les services et l'administration du port et arsenal de la marine à Rochefort, non daté (milieu du XVIII ${ }^{\mathrm{e}}$ siècle).

14. "Mais il y a beaucoup à travailler encore pour faire du port de Brest, un arcenal tout à fait commode ", Forfait, 1787, p. 204.

15. Kerfridin, Cros, 2011 ; Ortholan, Cros, Géhan, 2012. 
la Marine puisque l'ingénieur des bâtiments civils Pierre Toufaire équipe en 1783 le nouvel hôpital de l'arsenal de Rochefort d'une " pompe à feu " pour l'alimenter en eau au lendemain de la guerre d'Indépendance américaine ${ }^{16}$.

Les ordonnances de la marine de 1776 et de 1786 ont contribué à interpréter pleinement les arsenaux comme des espaces industriels où la rationalisation de l'implantation des bâtiments et la hiérarchisation des circulations concouraient à l'amélioration de leurs missions ${ }^{17}$. La meilleure illustration des progrès réalisés, et de ceux qui restent à venir, se trouve dans les plans d'aménagement levés sous l'impulsion du Maréchal de Castries entre 1784 et 1785 . Le ministre révèle l'ambition de ces plans dans une lettre adressée aux intendants et aux commandants le 20 mars 1784 où il indique que ces futurs documents contiendront tout ce qui doit « concourir à la meilleure disposition intérieure et à l'emplacement respectif de chaque objet pour former l'ensemble le plus avantageux à la sûreté, à la facilité, à l'économie et au bien du service de [chaque] département ${ }^{18}$ ». En outre, ces plans devront être accompagnés d'un mémoire qui indiquera " chaque partie d'améliorations nouvelles selon son degré d'urgence pour le service ${ }^{19}$ ». Cet élan ne se brise pas sur la Révolution et l'Empire. Selon leurs rythmes propres et dans un contexte très difficile, les arsenaux poursuivent leur modernisation et leur mécanisation. En s'attelant à la construction d'une réplique du moulin de Jean-Baptiste Hubert, ce projet patrimonial concourt de façon périphérique à déconstruire l'idée, encore très prégnante, d'une stagnation technique associée à la Révolution et à l'Empire, et à envisager une juste appréciation du travail des ingénieurs de la Marine au cours de cette période. Il permet aussi d'envisager la pluralité des voies techniques empruntées par ces hommes pour rationaliser leur espace de travail. Autrement dit, l'usage progressif de la machine à vapeur a lieu dans un contexte où l'on ne cesse de faire progresser les techniques de captation des énergies hydraulique et éolienne pour actionner différents mécanismes ${ }^{20}$. Cette coexistence et cette progression parallèle de plusieurs

16. Charpy, 2011.

17. Acerra, 1992, p. 179-189.

18. AN, Marine, $B^{2} 426$, f 255 , Lettre du Maréchal de Castries adressée aux arsenaux, 20 mars 1784.

19. AN, Marine, $B^{2} 426, f^{\circ} 255$.

20. Sur « l'accumulation incessante de ressources énergétiques anciennes et nouvelles » (p. 9), on se reportera à la très suggestive synthèse de François Jarrige et Alexis Vrignon qui n’hésitent pas à 
" systèmes techniques ${ }^{21}$ ", un constat désormais très classique ${ }^{22}$, ne sont pourtant pas si évidentes dans l'historiographie de la Marine française. Aussi, le plan de généralisation de la machine à vapeur dans les arsenaux, décidé par le ministre Pierre-Barthélémy Portal d'Albarèdes en 1818, est souvent interprété par les historiens comme le constat de leur retard et de leurs difficultés à entrer dans l'industrialisation ${ }^{23}$. L'idée selon laquelle la "vraie " mutation technologique des arsenaux a lieu sous Napoléon III est encore très prégnante, mais il s'agit à l'évidence d'un contresens et de l'impossibilité heuristique de considérer leur évolution sur le temps long.

\section{Jean-Baptiste Hubert et son moulin à draguer les vases}

Au début de l'Empire, Jean-Baptiste Hubert poursuit la mécanisation de l'arsenal de Rochefort en lui donnant un nouveau rythme, et en s'engageant aussi bien dans l'amélioration de machines mues par le vent et l'eau que par celles à vapeur pour lesquelles il devient un spécialiste au cours de la première moitié du XIX ${ }^{\mathrm{e}}$ siècle ${ }^{24}$. Il est né le $1^{\mathrm{er}}$ mai 1781 à Chauny, une ville qui borde le canal de l'Aisne, un environnement propice à éveiller chez le futur ingénieur le goût de la mécanique appliquée à l'eau qui caractérise toute sa carrière. En apprentissage quelque temps chez un horloger avec lequel il apprend la mécanique, Jean-Baptiste Hubert poursuit sa scolarité au collège de Soissons avant d'intégrer l'École Polytechnique en 1797 puis l'École des ingénieurs des vaisseaux en 1799, tout en suivant des cours

parler d'un « âge d'or des moulins à eau et à vent " pour le XIx ${ }^{\mathrm{e}}$ siècle : Jarrige, Vrignon (dir.), 2020, p. 47-50 ; toute aussi éclairante, l'étude de cas proposée par Didier Terrier, dans ce volume, consacrée aux moulins à vent pour faire de l'huile dans la région de Lille pendant la première moitié du XIX ${ }^{e}$ siècle qui fait écho aux choix techniques de Jean-Baptiste Hubert, "Moulins à vent à huile ", p. $57-70$.

21. Gille, 1979, p. 8-18.

22. Edgerton, 2013.

23. En outre, l'historiographie a souvent séparé artificiellement les arsenaux de marine des forges, fonderies ou manufactures implantées sur l'ensemble du territoire, des ensembles industriels sous l'autorité de la Marine et souvent les premiers à intégrer l'innovation technique (fonderies d'Indret, du Creusot, etc.).

24. Jean-Baptiste Hubert est l'un des ingénieurs de la Marine qui permet l'adoption de la propulsion à vapeur sur les navires de guerre. En 1822, il est membre de la première commission qui prépare la construction de remorqueurs à vapeur sur la Charente. Au cours de la période 1829-1840, il est le constructeur de tous les avisos et corvettes à vapeur de l'arsenal de Rochefort (Achéron, Eurotas, Sphinx, Fulton, Météore, Scamandre, Véloce et Caméléon) ; Brisou, 2001. 
à l'École des arts et métiers où il est élève de Jacques de Montgolfier ${ }^{25}$. Ingénieurs-constructeurs de la Marine en 1801, il poursuit sa formation à l'arsenal de Brest pendant trois ans, entrecoupée par un voyage en Hollande où il récolte de nombreux éléments sur les techniques liées aux moulins à vent. Nommé sous-ingénieur-constructeur de Rochefort en 1805, Hubert reste dans cet arsenal jusqu'à sa mort en $1845^{26}$.

Les sources pour aborder la trajectoire tant personnelle que professionnelle de Hubert sont peu abondantes. Le Service historique de la Défense à Rochefort rassemble l'essentiel de sa correspondance et les mémoires techniques qu'il a produits au cours de sa carrière dans cet arsenal. À la différence de bien d'autres ingénieurs, Hubert n'a pas publié de traités, à l'exception d'un Rapport sur les détails de construction des machines du bateau à vapeur le Sphinx en 1836 qui connaît une diffusion restreinte. Son projet d'édition d'une Application de la géométrie descriptive à l'architecture navale, en collaboration avec Jean Tupinier, son camarade de Polytechnique, est finalement abandonné. Néanmoins, toute la littérature grise destinée à faire part de son travail et de ses projets à ses collègues et au ministère de la Marine permet d'entrevoir la méthode de l'ingénieur pour innover. La mécanisation des tâches de l'arsenal procède d'une quantification préalable du travail humain nécessaire à la réalisation d'un produit ou d'un déplacement. Les gestes de l'ouvrier sont mesurés mathématiquement d'où découle ensuite la construction des machines destinées à aider le travail humain ou à en prendre le relais. La méthodologie de Jean-Baptiste Hubert s'inscrit dans le mouvement de rationalisation des opérations manuelles que l'on retrouve déjà au XviII ${ }^{\mathrm{e}}$ siècle avec le mouvement encyclopédiste ou dans certains corps d'ingénieurs, celui des Ponts et chaussées avec Jean-Rodolphe Perronet par exemple lorsqu'il s'agit d'améliorer les transports de matières premières sur les chantiers routiers ${ }^{27}$. En continuité avec l'Ancien régime, les arsenaux restent des lieux privilégiés de l'expérimentation savante et technicienne comme en témoigne le préambule

25. Brisou, 2001, p. 66.

26. Une fois en poste, les ingénieurs-constructeurs de la marine deviennent très peu mobiles, une caractéristique de ce corps et un paradoxe au regard des autres ingénieurs de l'époque. Entre 1805 et sa mort, Jean-Baptiste Hubert ne réalise qu'un détachement à Bordeaux entre 1812 et 1814 et un voyage en Angleterre en 1827 pour acheter, au nom de la Marine, une machine à vapeur. Cette faible mobilité physique est compensée par une correspondance riche avec des nombreux homologues, en Grande-Bretagne notamment.

27. Vacant, 2006. Picon, 1992. 
du rapport de l'ingénieur Charles Dupin consacré aux machines de JeanBaptiste Hubert en $1818^{28}$.

Hubert est à l'origine de très nombreuses machines mais le moulin à draguer la vase est très symbolique dans la mesure où il vient résoudre un problème récurrent depuis la création de l'arsenal en 1666, et qu'il s'agit de l'innovation qui propulse sa carrière d'ingénieur au service de la Marine ${ }^{29}$. Le projet est présenté à la fin de l'année 1805 puisque le préfet maritime fait écho de la démarche de l'ingénieur dans un courrier qu'il adresse le 12 frimaire an 14 (3 décembre 1805) au chef des constructions navales de l'arsenal de Rochefort, Jean-Denis Chevillard. Celui-ci envoie le 20 janvier 1806 un mémoire à Denis Decrès, le ministre de la Marine, pour lui rendre compte du mécanisme et du mouvement de la drague. Dans une période de mobilisation militaire aussi intense, l'obstruction de la double forme handicape le programme de construction navale de l'arsenal. Au moment où est discuté le projet de Hubert, le préfet maritime ordonne un nouveau " dragage par bœufs " afin de faire sortir deux bâtiments en cours d'achèvement, tout en indiquant que le moulin supprimera ce désagrément ${ }^{30}$. L'approbation ministérielle pour l'exécution des travaux tombe

28. "Les arsenaux de la Marine, par les grands travaux dont ils sont le théâtre, par la précision des poids, des volumes, des surfaces, des contours, et par les degrés de dureté, d'élasticité ou de rigidité qu'il faut atteindre dans les produits de l'industrie, exigent le concours des moyens rigoureux que peuvent fournir les sciences physiques et mathématiques. Ces établissements offrent en même temps des secours très multipliés pour faire de belles expériences sur la force des matériaux, et sur l'emploi des machines destinées à les mouvoir, à les travailler et à les poser. ", Dupin, 1818, p. 939-950.

29. À la suite de cette construction, Jean-Baptiste Hubert est proposé à l'avancement pour devenir « ingénieur ordinaire " par Jean-Denis Chevillard, le chef des constructions navales : "J'ai l'honneur de vous prévenir que la machine inventée par $\mathrm{Mr}$ Hubert [...] ayant obtenu un succès complet, [...] Mr le préfet maritime a cru dans sa justice devoir demander pour lui le grade d'ingénieur. », Service historique de la Défense [désormais SHD], Marine, Rochefort, fonds Hubert, 1 S 11, 12 février 1807.

30. "J'ai l'honneur de vous annoncer que je viens de faire fonctionner le dragage pour enlever les vases qui obstruent l'entrée du double bassin de construction. Les deux bâtiments que nous avons à sortir du bassin, le vaisseau l'Ajax et le brick le Griffon, seront terminés pour être mis à l'eau aux marées de mai. [...] La machine inventée par Mr Hubert nous mettra à même de supprimer la drague à l'avenir [...] Il aurait été avantageux et économique d'appliquer cette machine aux travaux que nous faisons maintenant avec l'ancien procédé. Les deux bâtiments que nous avons à sortir du bassin auraient été retardés jusqu'au mois d'août ou septembre si j'avais attendu l'établissement du moulin de Mr Hubert pour draguer la vase. J'ai préféré faire la dépense d'un dragage par bœufs pour accélérer l'armement du vaisseau et du brick. ", SHD, Marine, Rochefort, 2A4, le préfet maritime au ministre de la Marine, 22 mars 1806. 
le 3 mars 1806 et la solution technique proposée par Jean-Baptiste Hubert est à la fois simple et inattendue. Dans son projet, il propose de tendre un câble dans l'axe de la forme sur lequel est fixée une petite embarcation équipée d'une pelle mobile et faisant des va-et-vient (Fig. 1). Lorsque la pelle est abaissée, le bateau recule et la vase est évacuée dans le courant de la Charente. La pelle est alors levée et la drague repart dans l'autre sens.

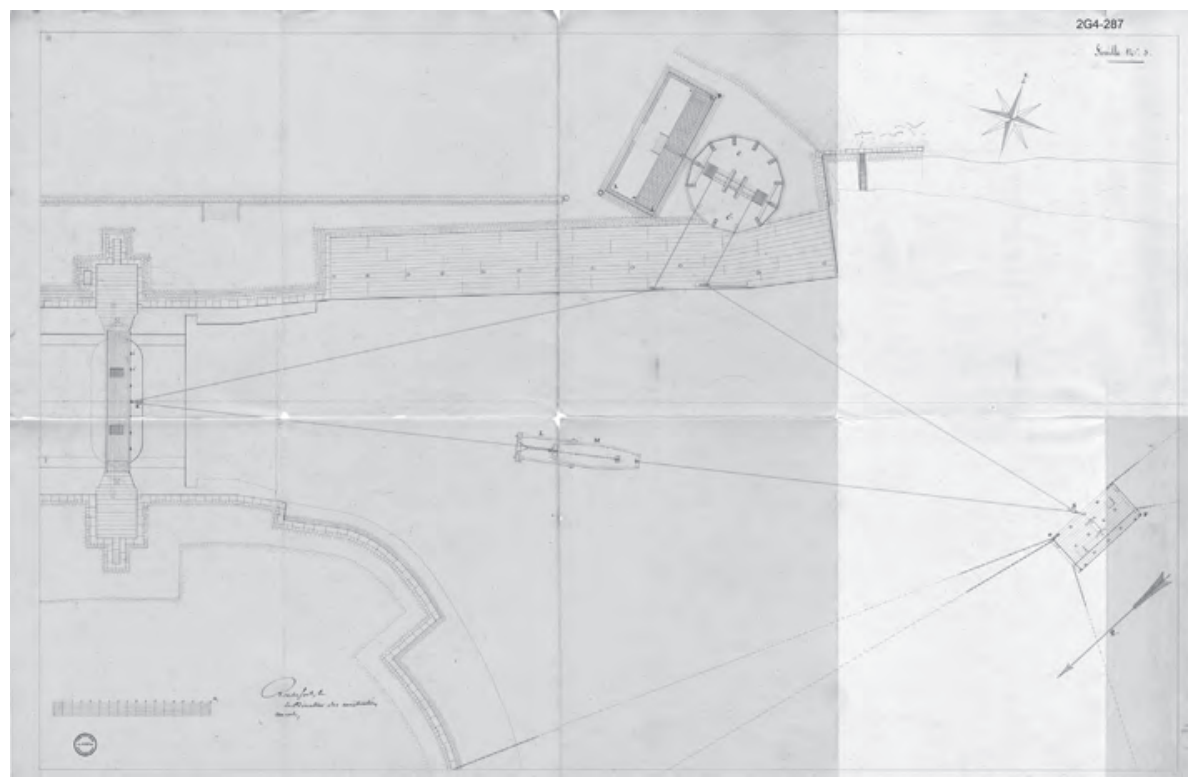

\section{Fig. 1. - Plan de dragage devant l'entrée de la double forme de radoub}

Service historique de la Défense, Marine, Rochefort, 2 G ${ }^{4}$ 287-1503 (2)

Le principal problème réside dans l'énergie nécessaire au déplacement de cette embarcation, d'autant plus que les vases transportées sont très lourdes (Fig. 2). Larsenal de Rochefort est installé sur un site quasiment sans énergie, les ressources hydrauliques étant très limitées. Dans ce paysage très plat, le moulin à vent s'impose. Le 28 janvier 1807, la commission chargée de l'examen du nouveau système de dragage rend un avis très positif au ministre :

La commission [...] n'hésite pas à penser que le moulin est un établissement extrêmement avantageux sous le rapport de la célérité et pour celui de l'économie en hommes et en argent, parce qu'on pourra désormais entrer dans les bassins des nouvelles formes à toutes les marées, les bâtiments qu'on était autrefois obligé de 
caréner sur un ponton et qu'on fera par conséquent en 24 heures avec beaucoup moins de dépenses, les travaux qui demandaient six jours sur rivière. L'avantage deviendra encore plus grand lorsque les bâtiments exigeront des réparations dans leur carène. Il fallait à flot virer les bâtiments des deux côtés, on ne pouvait donc y placer que la moitié des ouvriers qu'on pourra y occuper lorsqu'ils seront à sec dans les bassins qui d'ailleurs étant couverts ôterons aux ouvriers dans les saisons rigoureuses tout prétexte pour ne pas travailler d'où dérive nécessairement l'économie et surtout une célérité incalculable dans les opérations. La commission doit en terminant son rapport rendre hommage aux talents de M. Hubert, officier du génie maritime qui est l'inventeur de cette machine. Le mécanisme en est infiniment ingénieux, il remplit parfaitement le but qu'il s'est proposé par son exécution est à l'abri de toute critique ${ }^{31}$.

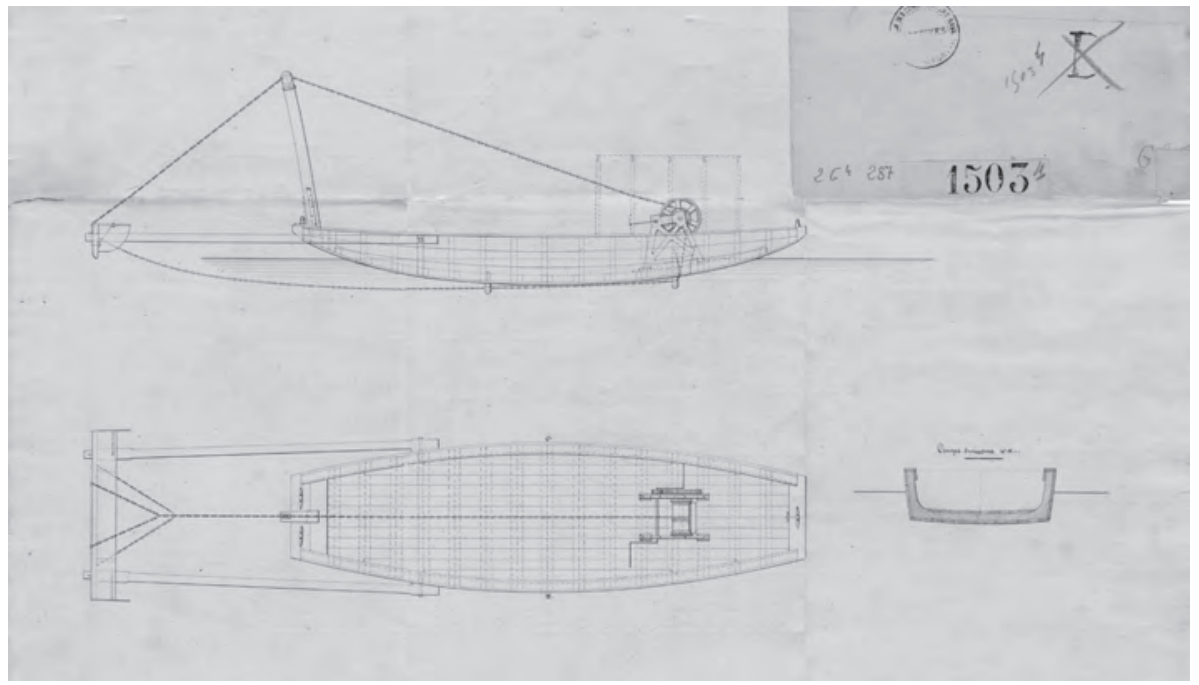

\section{Fig. 2. - Plan et coupe du bateau racleur}

Service historique de la Défense, Marine, Rochefort, 2 G ${ }^{4}$ 287-1503 (4)

Les travaux de construction du moulin s'élèvent alors à 26389 francs et 64 centimes quand il était nécessaire de débourser près de 20000 francs à chaque dragage par les bœufs ${ }^{32}$. Avec sa capacité de 257 coups de drague par 24 heures, le moulin peut être utilisé seulement un à deux jours par 
semaine pour évacuer efficacement les vases à l'entrée de la forme. Un laminoir à plomb, un broyeur à pigments de six meules et un tour à métaux sont ajoutés dans les étages du moulin afin de profiter de l'énergie disponible quand celui-ci n'est pas utilisé à draguer la vase. L'innovation technologique ne réside pas tant dans l'utilisation de l'énergie éolienne, on retrouve ce procédé dans d'autres arsenaux en France et en Europe, que dans la combinaison du système propulsif et du système de drague.

\section{L'évolution technique du moulin au $\mathrm{XIX}^{\mathrm{e}}$ siècle}

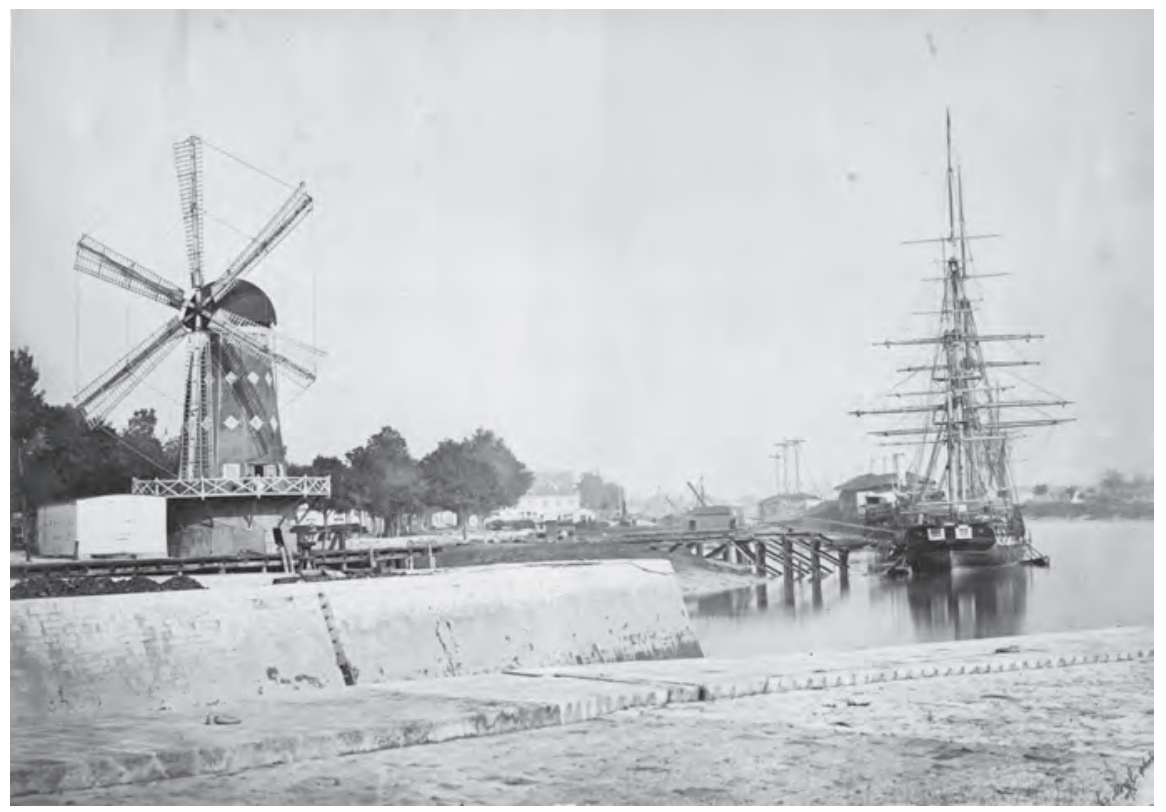

\section{Fig. 3. - Photographie du moulin en novembre 1866}

Service historique de la Défense, Marine, Rochefort, 2 G 908 (318)

Ce moulin s'impose dans le paysage de l'arsenal et de la ville de Rochefort, jusqu'à devenir un point de mire pour les habitants et les visiteurs de passage $^{33}$. En dépit de l'adoption progressive de la machine à vapeur, le moulin est conservé et poursuit sa mission jusque dans les années 1860 (Fig. 3). Faire le choix de la machine à vapeur doit être pertinent d'un point de vue

33. Voir le poème lu à la Société de Littérature, Sciences et Arts de Rochefort au cours de la séance du 22 mai 1810, cf. annexe. 
technique et rentable économiquement. Entre 1820 et les années 1840, les arsenaux s'en équipent sans nécessairement la généraliser. Le pompage de l'eau des formes de radoub et les forges adoptent rapidement la machine à vapeur tandis que les moulins à vent sont sans cesse améliorés pour le sciage des bois comme pour le dragage de la Charente. Les arsenaux et les ports européens sont loin d'avoir abandonné l'usage de l'énergie éolienne et la circulation des savoirs relatifs aux moulins se poursuit à l'ère de l'industrialisation. Entretenant des contacts étroits avec ses homologues anglais, Jean-Baptiste Hubert propose de changer les arbres en bois du moulin par de nouveaux en fer en prenant l'exemple du port de Liverpool :

On sait que les arbres en bois qui reçoivent des ailes des moulins à vent tournent sur une partie qui est arrondie et garnie de lames de fer pour former un tourillon d'un diamètre égal à l'équarrissage de ces arbres; il en résulte un frottement qui consume à pur perte une partie de la force motrice. Cette observation n'a pas échappé aux Anglais et, depuis longtemps, ils font usage d'arbres en fer dans la plupart des nombreux moulins à vent que l'on remarque même dans les villes telles que Liverpool où l'on fait usage des machines à vapeur. Ils ont aussi adopté des procédés mécaniques qui augmentent ou diminuent la surface des voiles de manière à régulariser la vitesse de ces machines et les ailes sont orientées par l'action même du vent. Je ne propose pas de mettre immédiatement en usage tous ces perfectionnements, mais je m'arrêterai à ce qu'il y a de plus essentiel en espérant le désir qu'il soit confectionné aux forges de Guérigny deux arbres en fer destinés à remplacer les arbres en bois des moulins à scier et à draguer. Ces pièces qui servent depuis longtemps ne peuvent avoir une longue durée ${ }^{34}$.

Jusqu'aux années 1850, il n'y a guère d'alternative au moulin à vent, même si son concepteur évoque, à la fin de sa carrière, son remplacement par une machine à vapeur afin d'améliorer la vitesse et la quantité de vase déplacée $^{35}$. Ses réflexions s'insèrent d'ailleurs dans un moment où la vapeur s'impose progressivement aux travaux de dragage pour en améliorer le rendement. En 1844, le mécanicien A. Castor met au point la drague à godet

34. SHD, Marine, Rochefort, $1 \mathrm{G}^{2}$ 62, Mémoire sur le service de la direction des constructions navales, Concernant les moulins à scier et à draguer, Jean-Baptiste Hubert, 25 juillet 1831.

35. SHD, Marine, Rochefort, $1 \mathrm{G}^{2}$ 63, 1832. 
et à vapeur sur la Seine ${ }^{36}$. Ce principe technique ne cesse d'être amélioré ensuite, notamment au moment du percement du canal de Suez ${ }^{37}$. Mais à Rochefort, les premiers projets d'une drague à vapeur sont présentés en 1859 , à un moment où l'arsenal s'équipe d'un bassin neuf précédé par un chenal de 80 mètres. Dans ce nouveau contexte, le moulin n'est plus suffisant et une drague manuelle assurée par des hommes munis de pelles est nécessaire en complément pour obtenir un lit de rivière utilisable ${ }^{38}$.

Le moulin disparaît à la suite de deux innovations techniques qui ont comme point commun d'avoir été proposées par des ingénieurs polytechniciens tous spécialistes des machines à vapeur. Le 29 avril 1859, c'est d'abord la question de la propulsion du bateau racleur qui est remise en question à la suite d'un mémoire de l'ingénieur Joseph-Émile Joëssel qui envisage de l'équiper d'une machine à vapeur de 6 chevaux permettant jusqu'à six allers-retours par heure. Les premiers essais sont suffisamment pertinents pour conduire la Marine à conclure un marché avec l'entrepreneur Ernest-Alexandre Goüin le 9 janvier 1860 pour passer définitivement à une drague à vapeur ${ }^{39}$. Pourtant, le procédé ne donne pas entière satisfaction et c'est le principe même du mouvement alternatif qui est remis en question. Afin de se débarrasser d'un plus grand volume de vase, l'ingénieur Émile Victor Courbebaisse envisage de rendre la drague plus mobile et de l'équiper d'un tuyau métallique muni d'une petite hélice à son entrée aspirant la vase. Ce nouvel outil est présenté à l'Exposition universelle de 1867

36. Note succincte sur les travaux exécutés depuis 1842 jusqu’à nos jours par A. Castor, en collaboration avec M. Hersent depuis 1856, Paris, 1873, cité par Barjot, 2006, p. 82.

37. Montel, 1995.

38. Le 29 avril 1859, le directeur des constructions navales de l'arsenal, Joffre, indique au préfet maritime que " le bassin neuf devant être bientôt terminé, nous avons besoin d'un appareil de dragage pour enlever la vase qui se déposera dans le chenal qui doit y conduire. L'ancien bassin est dragué au moyen d'un appareil dont le moteur est un moulin à vent mais il est souvent arrivé que par faute de vent cet appareil n'a pas pu fonctionner et alors, on était contraint de suppléer à cette machine au moyen de dragues manœuvrées à bras. J'ai pensé qu'il convenait de donner le mouvement au moyen d'une machine à vapeur parce que nous serons libres de l'employer toutes les fois que le besoin l'exigera. ", SHD, Marine, Rochefort, $1 \mathrm{G}^{2}-205$, Joffre au préfet maritime, 29 avril 1859.

39. Ingénieur issu de l'École Polytechnique, Ernest-Alexandre Goüin est un entrepreneur important au XIX ${ }^{e}$ siècle, fondateur de la Société de construction des Batignolles spécialisée dans la mécanique, la métallurgie et les travaux publics. Décisif pour le développement du train à vapeur, Goüin est également un partenaire central pour la construction navale militaire à vapeur à partir des années 1850 , notamment après le rachat des chantiers Guibert à Nantes, un pionnier de la vapeur sur la façade atlantique depuis les années 1810-1820. Park, 2000, p. 361-386. 
et les premiers essais ont lieu à Rochefort le 12 juin 1871 avec un certain succès si l'on considère le volume des vases déplacé (cf. tableau 1). Cette double évolution condamne à l'obsolescence le moulin de Jean-Baptiste Hubert mais les archives disponibles sont muettes quant au moment de sa destruction (Fig. II, cahier couleur) ${ }^{40}$.

Tableau 1 - Comparaison des différentes solutions de dragage des vases établie en 1871

\begin{tabular}{|l|l|l|l|}
\hline \multicolumn{1}{|c|}{ Nature de la drague } & \multicolumn{1}{c|}{ Manuelle } & \multicolumn{1}{c|}{ À vapeur } & \multicolumn{1}{c|}{ À hélice } \\
\hline $\begin{array}{l}\text { Tirant d'eau } \\
\text { de l'embarcation (mètre) }\end{array}$ & 0,55 & 0,63 & 0,60 \\
\hline $\begin{array}{l}\text { Prix de revient (moteur } \\
\text { compris le cas échéant) }\end{array}$ & 2560 & 32500 & 8254 \\
\hline Nombre d'hommes & 6 & 7 & 5 \\
\hline Salaire journalier (francs) & 13,4 & 15,9 & 11,5 \\
\hline $\begin{array}{l}\text { Volume de vase dragué } \\
\text { en } 10 \text { heures et transporté } \\
\text { de } 60 \text { mètres (en } \mathrm{m}^{3} \text { ) }\end{array}$ & 37,5 & 126,9 & 1500 \\
\hline
\end{tabular}

Source : SHD, Marine, Rochefort, $1 \mathrm{G}^{2}$ 207, Note relative aux essais d'un nouvel appareil dragueur à outil mobile, Émile Victor Courbebaisse, 12 juin 1871.

\section{Les enjeux de la restitution du moulin à draguer}

La contextualisation de l'apparition et du fonctionnement, tout au long du $\mathrm{XIX}^{\mathrm{e}}$ siècle, du moulin de Jean-Baptiste Hubert, a été l'étape préalable au rassemblement des forces - associative, politique et économique - susceptibles de soutenir cette restitution, et à la commande des études techniques validant ou non sa faisabilité. Mais indépendamment de l'enthousiasme soulevé, dans le cadre d'un territoire familier avec ce type de projet depuis le chantier de l'Hermione, et de la volonté de donner une valeur heuristique à cet artefact technique, les débats quant à l'opportunité de sa réalisation ne manquent pas. Pratiquée depuis l'Antiquité, la restitution de monuments demeure une pratique qui ne fait pas l'unanimité, notamment en France où l'imaginaire collectif, et celui des spécialistes du patrimoine, l'associe

40. Léon Morel-Fatio peint sans doute le moulin dans les toutes dernières années de son utilisation. 
volontiers aux débordements de l'architecte Eugène Viollet-le-Duc au $\mathrm{XIX}^{\mathrm{e}}$ siècle. Au-delà des aspects techniques et financiers, l'un des enjeux réside donc dans la capacité à convaincre les pouvoirs publics, et notamment les représentants du ministère de la Culture, que cette restitution représente une plus-value pour l'interprétation d'un ensemble patrimonial plus vaste qu'est l'arsenal de Rochefort.

\section{Fédérer autour d'un projet patrimonial : brève histoire de l'Association du Moulin de l'Arsenal de Rochefort}

Commencée en 1997, la construction d'une réplique de l'Hermione, s'est achevée en 2012 pour ce qui concerne la coque, année de son lancement et de son transfert, de la double forme de radoub vers la forme Napoléon III, pour la mise en place de son gréement et de son armement. Cette opération a fait prendre conscience aux responsables de l'association Hermione-La Fayette et aux collectivités locales des contraintes imposées par la présence de la vase pour la manipulation des bateaux-portes fermant ces formes de radoub ${ }^{41}$. Dans ce contexte, plusieurs opérations ont dû être entreprises avant le lancement. Il s'agissait de s'informer de la disponibilité de la drague au moment où était envisagée la sortie de l'Hermione et d'établir un calendrier des travaux afin de dégager la double forme de radoub à temps, mais également prévoir le financement de ces opérations estimées à 150000 euros. Chaque mouvement de l'Hermione dans l'arsenal contraignait à reproduire le même processus et il a paru logique d'imaginer reprendre le système conçu par Jean-Baptiste Hubert, en répondant à des préoccupations contemporaines. La présence de la vase créée une situation de permanence forte qui place les responsables du site d'aujourd'hui devant les mêmes difficultés que ceux du Xvir ${ }^{\mathrm{e}}$ siècle. Or, le système de dragage et son moteur éolien reposent sur l'idée centrale que le meilleur des dragages est celui que l'on ne programme pas. Dans ce contexte, les

\footnotetext{
41. Les bateaux-portes ferment les formes de radoub : " Il y a certains bateaux qui servent ou qui sont construits pour fermer l'ouverture des bassins dans un port, \& cette fonction leur fait donner le nom de bateau-porte. Ils sont préparés de manière que leurs extrémités s'enchassent $\&$ descendent ou montent verticalement dans des coulisses qui bordent les ouvertures de ces bassins, selon que ces bateaux sont plus ou moins chargés ou déchargés. ", Romme, 1792, p. 74.
} 
solutions techniques d'hier pouvaient se révéler pertinentes et la construction d'une réplique du moulin avait un sens au XXI ${ }^{\mathrm{e}}$ siècle.

La première étape d'une réflexion sur l'intérêt et la faisabilité de la construction d'une restitution a lieu le 23 mai 2016 dans le cadre d'une réunion ouverte organisée au musée de la Marine de Rochefort. La forte audience suscitée par cette première rencontre révèle alors la volonté de la population locale de s'associer à ce projet patrimonial qui débouche sur la création de l'Association du Moulin de l'Arsenal de Rochefort (AMAR) à l'occasion d'une première assemblée générale le 22 septembre 2016, jour anniversaire du décès de Jean-Baptiste Hubert. L'AMAR se structure rapidement en quatre groupes interdépendants destinés à alimenter les différentes phases du projet. Le groupe histoire recense toutes les sources relatives au moulin et au travail de Jean-Baptiste Hubert. Dans ce contexte, une convention est signée avec le Service Historique de la Défense qui conserve l'essentiel de la documentation, à Rochefort et à Vincennes. Celle-ci est composée des mémoires de l'ingénieur, des rapports d'expertise et d'inspection avant et après la construction, ainsi que d'un ensemble iconographique composé de plans, de croquis et de représentations de l'arsenal où le moulin est en évidence. Cette valorisation des sources manuscrites et iconographiques a été menée simultanément à une prise de cotes sur les maquettes réalisées en 1806 et en 1829 exposées au musée de la Marine de Rochefort (Fig. III et IV, cahier couleur). Ce corpus a permis de préciser les principes techniques du système de drague mais également d'appréhender son fonctionnement quotidien avec ses pannes et ses améliorations tout au long du $\mathrm{XIX}^{\mathrm{e}}$ siècle. Ces sources alimenteront la mise en œuvre d'outils de communication à destination du public mais il s'agit également d'envisager leur diffusion auprès de la communauté scientifique selon des modalités qui ne sont pas encore arrêtées.

L'ensemble des informations collectées est exploité par le groupe technique qui conduit plusieurs études in situ afin d'évaluer l'emplacement du futur moulin et les contraintes de sa conception. Pour cet aspect, l'AMAR a reçu un soutien important avec la Fédération des Moulins de France et la Fondation Arts et Métiers qui a envoyé à Rochefort des élèves ingénieurs de l'ENSAM de Cluny. Il s'agissait de comprendre le fonctionnement du bateau racleur mais également d'étendre son action. En 1805, seul le dragage de la double forme est pensé mais il s'agit aujourd'hui d'inclure également la forme Napoléon III. Or, il existe une distance de 110 mètres 
entre les deux chenaux de la double forme et du bassin Napoléon III et ce dernier a un seuil plus profond d'environ 1,80 mètre, autant de contraintes techniques qu'il s'agit de dépasser dans le futur projet. Un troisième groupe est plus spécifiquement chargé de la communication et de la recherche de partenaires financiers. Ses membres se sont attachés à établir différents moyens de communication (logo, dépliants, lettres d'information, site internet, etc.) afin de donner une visibilité au moulin lors de sa phase de lancement. Enfin, un groupe d'exploitation touristique a pour mission d'anticiper au mieux les besoins qui devront être satisfaits pour recevoir du public pendant et après la construction des éléments qui constituent l'ensemble du projet, en considérant que la réalisation prévue sera une des composantes du site de l'arsenal de Rochefort.

Ce projet patrimonial s'est en effet très vite intégré dans une démarche globale de requalification de l'arsenal de Rochefort à la recherche d'une nouvelle dynamique de développement économique et touristique après la fin du chantier de l'Hermione. Face à un ralentissement notable de la fréquentation du site, les collectivités locales - ville de Rochefort, Communauté d'Agglomérations Rochefort Océan, Conseil départemental de la Charente Maritime, Conseil régional de la Nouvelle Aquitaine - commandent en 2017 une expertise auprès de Médiéval-AFDP, une agence-conseil spécialisée dans la mise en valeur des patrimoines et des territoires. Dans son rapport conclusif, la restitution du moulin de Jean-Baptiste Hubert est mentionnée comme l'un des travaux innovants cumulant les atouts historique, touristique, économique et écologique. Dans ce contexte, l'AMAR rejoint les différents acteurs du site pour l'obtention du label Grand Site pour l'arsenal mais également les rives et l'estuaire de la Charente ${ }^{42}$.

\section{Les atouts du projet}

D'un point de vue historique, la reconstruction de ce moulin a l'ambition d'apporter une grille de lecture complète de l'arsenal de Rochefort.

\footnotetext{
42. Le label Grand Site est octroyé par le ministère de l'Environnement à un site très fréquenté qui s'engage à la "protection des monuments naturels et des sites de caractère artistique, historique, scientifique, légendaire ou pittoresque " (loi n ${ }^{\circ}$ 1930-05-02 du 2 mai 1930 reprise dans la loi $\mathrm{n}^{\circ}$ 2010-788 du 12 juillet 2010, article 150 du code de l'Environnement). Le projet de l'AMAR rejoint également la nouvelle " marque » créée en 2019, "L'Arsenal des Mers ", qui consiste à fédérer les quatre sites de l'arsenal (Corderie, Accro-mâts, Hermione et Musée national de la Marine) dans une nouvelle offre touristique.
} 
Actuellement, il y a peu d'infrastructures spécifiques au XIX ${ }^{\mathrm{e}}$ siècle et qui remettent en perspective le rapport du site avec le fleuve tout en montrant la volonté de l'État de faire face à un environnement contraignant en choisissant des solutions techniques adaptées. Pour faire écho à la partie précédente, il s'agit aussi d'envisager la période $1760-\mathrm{XIX}^{\mathrm{e}}$ siècle, comme un moment clé pour les arsenaux européens et plus particulièrement celui de Rochefort qui, à l'échelle de la France, est l'infrastructure militaire qui a souvent été à l'avant-garde de l'innovation. Il s'agit de valoriser cette séquence particulière de l'histoire des techniques en l'attachant à son promoteur principal, Jean-Baptiste Hubert, issu des premières promotions de l'École Polytechnique et qui s'emploie non seulement à mécaniser l'arsenal, notamment avec le moulin, mais également à faire entrer la Marine française dans l'ère de la propulsion à vapeur. Cet éclairage sur le XIX ${ }^{e}$ siècle et l'ère industrielle aboutit à la construction d'une histoire " complète " du site actuel et autorise conséquemment la construction d'une médiation patrimoniale beaucoup plus cohérente ${ }^{43}$. Ce nouveau discours et la monumentalité du moulin débouchent sur le renforcement de l'attractivité touristique du site, là encore en lui apportant un surcroît de cohérence, mais liée à son appréhension spatiale cette fois. Culminant à une trentaine de mètres au-dessus de la Charente, le moulin représentera un lieu d'interprétation de l'ensemble de l'espace alentour en proposant au public d'envisager d'un coup d'œil la configuration spécifique d'un arsenal de rivière, son inscription urbaine et environnementale. En outre, il ne faut pas négliger l'impact du moulin dans la structuration des flux de visiteurs au sein de l'arsenal. Au niveau local, le moulin sera un trait d'union entre la corderie, dont l'attractivité est très forte, les formes de radoub et le musée de la Marine. Dans le contexte spatial de Rochefort, toutes les infrastructures sont implantées le long de la Charente et le visiteur a besoin d'un fil d'Ariane pour les relier les unes aux autres. En terminant sa découverte de la corderie, le visiteur sera conduit à la principale zone de construction

43. Le manque de médiation patrimoniale autour du XIX ${ }^{e}$ siècle est lié à la faiblesse de l'historiographie des arsenaux de cette période, en France comme à l'étranger. L'important renouvellement de la recherche concernant l'histoire des marines de guerre sous l'Ancien Régime à partir des années 1970 a servi de matrice à la valorisation patrimoniale de la corderie ou des formes de radoub et a sans doute conduit à mythifier ce moment dans l'imaginaire collectif, pour preuve le choix de la reconstruction de l'Hermione et pas d'un navire à vapeur par exemple, alors qu'il s'agit aussi d'un marqueur fort de l'activité de l'arsenal au $\mathrm{XIX}^{\mathrm{e}}$ siècle. Pour une remise en perspective de cette historiographie et de son usage : Cabantous, 2001, p. 33-43 ; Llinarès, Hrodej, 2010, p. 317-339. 
de l'arsenal - où il découvrira les formes de radoub et l'Hermione - via ce repère visuel. Enfin, avec sa silhouette visible de très loin, le moulin renforcera l'identité originale de Rochefort dans le plat pays rochefortais.

Si les arguments historiques et le rayonnement culturel représentent des préoccupations majeures, les projets de cette nature doivent prendre en compte des problématiques économiques couplées à une vigilance écologique et à la notion de durabilité, des éléments souvent décisifs pour les pouvoirs publics et les financeurs. L'une des forces de cette restitution est sa capacité à lier l'ensemble de ces intérêts. Prosaïquement, la réintroduction de ce moulin aux abords des formes de radoub supprimera le coût de dragage généré lors de chaque ouverture des bateaux-portes. L'accès permanent aux différents bassins de cette zone a un double intérêt puisqu'il permet l'entrée et la sortie de l'Hermione sans difficultés et qu'il autorise une requalification économique de cet ensemble patrimonial en projetant la mise en place d'un chantier dit de "refit ", un secteur très porteur sur cette partie de la côte atlantique ${ }^{44}$. Dans l'ensemble projeté, cette activité contemporaine est porteuse de sens pour envisager une compréhension globale du rôle d'un arsenal de la marine, le refit faisant inévitablement écho au rôle d'entretien et de réparation des formes de radoub depuis le $\mathrm{XVII}^{\mathrm{e}}$ siècle. À la croisée des préoccupations économiques et écologiques, la captation de l'énergie éolienne sera destinée à fournir l'électricité nécessaire au fonctionnement du bateau racleur mais également aux différents besoins de l'arsenal. L'étude consacrée à l'ingénierie du moulin, menée par les élèves-ingénieurs de l'ENSAM, a mis en évidence que le bateau racleur consommerait 1,5 MWh/an sur les 13,5 MWh/an produit par le moulin. Il conviendra d'étudier quelles sont les possibilités d'utilisation, sur le site de l'arsenal, de l'énergie fournie par le moulin, simultanément ou non avec le fonctionnement du bateau racleur. Enfin, en assurant un dragage des vases de façon quotidienne lors des marées descendantes, il apparaît que la perturbation de l'écosystème de la Charente est minimale au regard d'un curage massif une à deux fois par an. Tous ces éléments font de la reconstruction de ce moulin et du bateau racleur une expérience patrimoniale

44. Le " refit ", littéralement le " retapage ", concerne toutes les techniques d'entretien et de transformation des navires anciens (charpenterie navale, mécanique, voilerie, gréements, ébénisterie, chaudronnerie, sellerie, etc.). Dans un contexte de crise de la construction navale en Europe à la fin du $\mathrm{Xx}^{\mathrm{e}}$ siècle, de nombreux chantiers sont passés de la construction au refit en développant un savoir-faire très qualitatif (La Rochelle, La Seyne-sur-Mer, La Ciotat, etc.) 
novatrice où les enjeux économiques et environnementaux occupent une place centrale. En ayant un impact quasiment nul sur l'environnement et en produisant une énergie " propre ", ce moulin acquiert une dimension écologique fondamentale qui a été un argument décisif pour entraîner certains décideurs pas toujours sensibles à la dimension historique du projet ${ }^{45}$.

\section{Conduire un projet de restitution patrimoniale dans un environnement protégé}

S'appuyant sur l'expérience acquise de mise en valeur de l'arsenal de Rochefort, et notamment le chantier de l'Hermione, l'AMAR, dont de nombreux membres ont été partie prenante des initiatives précédentes, est parvenue à élaborer un argumentaire et une méthodologie pour mener à son terme cette entreprise patrimoniale inédite. Néanmoins, si ce projet de restitution semble fédérateur, son inscription spatiale, sur un territoire sensible et protégé, ainsi que la nature même de l'objet attendu contraignent ses promoteurs à négocier en permanence sa mise en œuvre.

L'arsenal de Rochefort est au cœur d'un réseau de protections, amplement justifié par la richesse et la fragilité du site que son histoire patrimoniale permet de remettre en perspective. En août 1944, les troupes d'occupation allemandes incendient les bâtiments de l'arsenal et sabordent des navires afin d'interdire l'accès au port. La corderie, emblème du site depuis sa création en 1666, brûle pendant plusieurs jours. L'espace qui correspond aujourd'hui au cœur touristique de la ville demeure une friche durant plus de 30 ans, témoin à la fois de l'occupation - Rochefort n'était pas en zone libre - et de la perte irrémédiable de la principale activité économique de la région depuis la fermeture de l'arsenal en 1927. C'est aussi un passé porteur de l'identité de la ville qui disparaît lentement au milieu des décombres et de la végétation ${ }^{46}$. Les années d'après-guerre sont pour Rochefort celles d'une cité sombre, réputée, non sans raison, sale et violente, où les troupes françaises et américaines en garnison se retrouvent dans d'innombrables débits de boissons et de très actifs quartiers de prostitution. Il n'est guère question de tourisme, de culture ou de patrimoine.

45. Gasnier, 2011, 2018 et 2019, p. 339-346.

46. Ponsonnet (dir.), 2008 ; Dupont, Fardet, 1993. 
L'intérêt de l'Amiral Maurice Dupont, commandant de l'arrondissement maritime de Rochefort-La Pallice entre 1964 et 1967, redonne un temps un avenir à la corderie. Il fait partiellement défricher les ruines par les appelés du contingent et l'ensemble est classé au titre des monuments historiques grâce à son intervention et à celle de Jean Verney, alors directeur du Comité régional des affaires culturelles (CRAC) de Poitou-Charentes ${ }^{47}$. En 1966, Les Demoiselles de Rochefort, que Jacques Demy tourne en plein centre-ville et où de nombreux Rochefortais sont figurants, change la perception que les habitants ont de leur propre ville : la culture y a sa place et le film a joué un rôle majeur dans cette prise de conscience sans laquelle il n'y a pas de patrimoine. En 1971, alors que l'État porte un projet de route à quatre voies passant entre la corderie et la Charente, l'équipe municipale de Jean Morin s'y oppose et, sous l'impulsion de son élu aux affaires économiques Jean-Louis Frot, engage le développement de Rochefort dans une idée de rénovation de son patrimoine bâti. Profitant d'un dispositif d'aide de l'État aux villes moyennes, les travaux débutent en 1974, avec la corderie en fer de lance. Ils durent vingt ans et l'objectif est une réappropriation de leur passé par les habitants et la construction d'une identité commune. Les abords du fleuve sont réintégrés à la ville et l'objet d'un aménagement paysager contemporain à l'issue d'un concours remporté par Bernard Lassus qui met en place le Jardin des Retours, inauguré en 1991. L'Hermione, dont le chantier démarre en 1997, suit très logiquement ce mouvement de longue durée.

Le projet de restitution du moulin Hubert s'inscrit dans un espace qui est à la fois ancien et récent, constitué d'un bâti dont l'apparente solidité ne peut faire oublier qu'il a failli disparaitre, une première fois en 1944, une deuxième il y a quarante ans. L'action politique, déterminante dans le sauvetage et la valorisation de cet ensemble patrimonial, s'est logiquement accompagnée d'un ensemble de protections soucieuses de conférer au site sa pérennité et son intégrité, autant sur le plan des monuments historiques que par rapport au paysage. Ainsi, l'espace de l'arsenal regroupe plusieurs édifices protégés au titre des monuments historiques ${ }^{48}$, dont l'entretien et

47. Par arrêté du 10 octobre 1967, la corderie, ainsi que ses bâtiments annexes (château d'eau, fontaine et corps de garde), est classée au titre des monuments historiques.

48. Outre la corderie, la porte dite porte du soleil est classée monument historique par arrêté du 11 juin 1928, l'Hôtel de Cheusses, qui abrite le musée de la Marine, le 19 janvier 1932, la forme Napoléon III, qui sert de mouillage à l'Hermione, le 7 avril 1988, la double forme de radoub le 9 mai 
les modifications sont soumis au contrôle de l'État, et la commune de Rochefort a décidé de mettre en place un secteur sauvegardé en 2017. Le Plan de sauvegarde et de mise en valeur (PSMV) qui l'accompagne vise à tenir compte des contraintes proprement patrimoniales dans le développement urbain. L'évolution de la ville se fait dans ce cadre en tenant compte des éléments remarquables d'un point de vue historique et esthétique, y compris à l'intérieur des immeubles. À Rochefort, l'arsenal fait naturellement partie du secteur sauvegardé et toute modification de l'espace, comme c'est le cas avec la restitution du moulin Hubert, est soumise à l'Architecte des bâtiments de France.

Ce cadre contraint du PSMV sera renforcé par celui du label Grand site ${ }^{49}$ qui a déjà conduit dans un premier temps au classement de l'estuaire de la Charente en 2013, au titre de la loi du 2 mai 1930. Ce dispositif complémentaire fait du paysage un élément patrimonial en tant que tel, qu'il convient de maintenir en l'état. Les activités qui s'y déroulent sont à la fois conditionnées par la recherche d'un équilibre, notamment pour éviter de le dégrader, mais également par l'ambition de garantir "l'esprit des lieux ", ouvrant le champ du patrimoine au domaine du sensible dont les contours doivent être définis en commun par les usagers du lieu.

\section{Quel statut patrimonial pour le moulin Hubert?}

Cette superposition de dispositifs réglementaires, qui vise à éviter toute modification significative du site et de son environnement, a d'emblée imposé aux porteurs de la restitution de trouver un interstice qui permettrait d'en justifier l'intérêt. En dépit de l'argumentaire développé par l'association, cette volonté s'est rapidement heurtée à la philosophie et à la déontologie qui sous-tendent ce corpus législatif dont l'Architecte des bâtiments de France en charge de Rochefort est le garant. Tout au long des études préparatoires à la construction du moulin, le dialogue entre le représentant du ministère de la Culture et ceux de l'AMAR a été franc

1989 et, enfin, l'Hôtel de la Marine, actuel Commandement des Écoles de la gendarmerie nationale, inscrit sur la liste des monuments historiques le 8 octobre 2015 (base Mérimée du ministère de la Culture consacrée au recensement du patrimoine architectural).

49. En juillet 2020, l'État a attribué ce label à l'estuaire de la Charente faisant de ce site le $21^{\mathrm{e}}$ à obtenir cette distinction. 
et ouvert sans dissimuler une opposition intellectuelle évidente. Pour lui, un monument historique, aussi documenté soit-il, ne peut être construit à l'identique sans être considéré comme un pastiche, une imitation. Cette copie apparaît comme une sorte d'imposture en donnant l'illusion d'une valeur patrimoniale qu'il ne possède pas. Un édifice ancien construit de matériaux neufs est en somme un faux témoin qui livre du paysage une image recomposée, et de l'histoire un récit falsifié. En matière de monument historique, on ne peut envisager de reconstruire que ce qui est dégradé d'un édifice et de ce point de vue le projet du moulin n'entre pas dans cette catégorie. Ces principes ont été progressivement construits en réaction à certaines pratiques de restauration du $\mathrm{XIX}^{\mathrm{e}}$ siècle, dont celles d'Eugène Viollet-le-Duc sont sans doute les plus emblématiques, où il s'agit de compléter, recréer ce qui avait été détruit, quitte à proposer une vision totalement réinventée du passé et à achever ce qui ne l'avait jamais été. En réaction, l'architecte Camillo Boito (1836-1914) propose une voie médiane où toute intervention sur les vestiges et les monuments doit être visible pour éviter la confusion de l'ancien et du récent ${ }^{50}$, un principe qui guide la rédaction de la Charte d'Athènes de 1931 pour la restauration des monuments historiques. En 1964, la Charte de Venise la complète et pose notamment le principe du respect de la "substance " ancienne et affirme que la restauration "s'arrête là où commence l'hypothèse ${ }^{51}$ " (art. 9). En France, ce sont les restaurations conservatrices qui sont privilégiées et le principe de la restitution est en revanche souvent violemment critiqué, même s'il s'agit d'une pratique ancienne. En outre, il convient de préciser que ce positionnement de la France à l'égard du patrimoine ne fait pas consensus à l'échelle mondiale puisque la restitution peut apparaitre d'un usage courant dans d'autres cultures, notamment pour répondre à des enjeux identitaires et sociétaux. C'est le cas, par exemple, en Chine où le Temple du Ciel, détruit par un incendie en 1889, a été intégralement

\footnotetext{
50. Boito, 2000.

51. L'article 9 est le premier consacré à l'acte de restauration des monuments anciens dans la Charte de Venise : "La restauration est une opération qui doit garder un caractère exceptionnel. Elle a pour but de conserver et de révéler les valeurs esthétiques et historiques du monument et se fonde sur le respect de la substance ancienne et de documents authentiques. Elle s'arrête là où commence l'hypothèse, sur le plan des reconstitutions conjecturales, tout travail de complément reconnu indispensable pour raisons esthétiques ou techniques relève de la composition architecturale et portera la marque de notre temps. La restauration sera toujours précédée et accompagnée d'une étude archéologique et historique du monument. »
} 
reconstruit en 1998 et inscrit sur la liste du patrimoine de l'Unesco, d'autres exemples peuvent également être convoqués dans des pays ayant subi d'importantes destructions à la suite de conflits (pays d'Europe de l'Est, Japon, etc.).

Dans ce contexte, il apparaissait difficile de légitimer la construction d'un moulin dont il ne reste plus aucune trace visible sur le site de l'arsenal de Rochefort. Dès lors, comment concilier le cadre législatif existant avec l'idée même d'une restitution ? Paradoxalement, un premier argument se trouve peut-être au sein même de la Charte de Venise qui précise dès l'article $1^{\text {er }}$ que " La notion de monument historique comprend la création architecturale isolée aussi bien que le site urbain ou rural qui porte témoignage d'une civilisation particulière ». Le label Grand site qui fait du paysage un bien patrimonial est bien l'héritier de la Charte de Venise. Dans le cas du moulin, il est cohérent de considérer que le monument à restituer est l'espace de l'arsenal dans sa globalité, son paysage s'étant retrouvé amputé d'un élément utile à sa lisibilité. La restitution du moulin s'apparenterait alors à la réparation d'un accroc d'un tableau pour en retrouver l'intégrité et en améliorer la lecture par tous.

En outre, il a été nécessaire de s'accorder sur la nature de l'objet attendu. Les restitutions d'artefacts techniques dans une perspective scientifique et didactique ne sont pas encore très fréquentes. La reconstruction des petits objets mobiles est aisée $e^{52}$, mais lorsqu'il s'agit d'ensembles plus importants, les humanités numériques prennent le relais dans le cadre de projets de " rétroconception ${ }^{53}$ ". Les modélisations 3D sont l'objet de nombreux travaux stimulants et permettent d'envisager valorisation patrimoniale et médiation culturelle ${ }^{54}$. À Rochefort il s'agit d'aboutir à un " nouveau " moulin, mais cette restitution n'a pas l'ambition de reproduire ce qui n'est plus, mais plutôt de s'attacher à l'essentiel en s'autorisant des modifications qu'il ne s'agit pas de dissimuler, notamment en utilisant des techniques contemporaines. C'est une réalisation soucieuse de synthèse qui

52. Pour la reconstruction d'objets techniques nautiques, il faut mentionner les travaux récents de Céline Le Gall qui a fait reconstruire le cabestan et l'anémomètre du savant Giovanni Poleni : Le Gall, 2019, p. 433-464.

53. Cotte, 2009, p. 7-21. Dans ce domaine, il faut signaler les travaux menés actuellement dans le cadre du projet ANR ReSeed "Rétro-conception sémantique d'objets patrimoniaux digitaux » de Florent Laroche, Alain Bernard, Loïc Jeanson et Paul François à l'École Centrale de Nantes.

54. Voir le cas des arsenaux de Brest et de Venise : Abiven, 2019. 
travaille sur les lignes de force d'un édifice technique. Ici, c'est le principe du dévasement par la force éolienne qui est important car c'est ce qui est significatif de l'histoire de Rochefort. Cette technique raconte l'effort pour composer avec l'environnement et innover sous la contrainte pendant près de trois siècles, et témoigne in fine d'une part fondamentale de l'esprit des lieux. Cette restitution fonde sa légitimité sur cet art de la synthèse et sur sa capacité à raconter l'histoire au plus grand nombre. Le moulin ne dénature pas les éléments anciens encore en place sur le site mais il tend au contraire vers la construction d'une nouvelle authenticité globale de l'arsenal de Rochefort $^{55}$. À l'échelle du moulin, sa nature d'objet de médiation autorisera des manipulations, des expérimentations et apparaitra ainsi comme un moyen d'approcher les gestes des hommes de l'époque et de raconter l'histoire des techniques. Un parallèle peut être réalisé avec les restitutions des grottes préhistoriques qui posent des difficultés voisines quant aux valeurs patrimoniale et d'authenticité ${ }^{56}$. Dans le cas de Lascaux II, l'historienne des sciences et spécialiste des représentations de la préhistoire, Claudine Cohen, indique

Les techniciens et les artistes auteurs de Lascaux II nous révèlent que la fabrication d'un "faux " n'est pas seulement mécanique. Elle exige une communion profonde, par le geste même, avec les hommes de la préhistoire. L'étude détaillée, analytique (et non pas synthétique comme est le regard qui admire ou la photographie), des techniques, des tracés, des couleurs, des formes, de l'utilisation du support, fait pénétrer dans la pensée même du peintre, en tant qu'il est à la fois artiste et porteur d'un savoir technique et d'une sensibilité particulière. Le pastiche, disait Proust, est peut-être la forme idéale de la critique, car il est une tentative de comprendre et de retrouver la vision qui a donné naissance à l'œuvre. La recréation d'une œuvre à travers le pastiche ou le « faux " met en lumière les procédures même de sa constitution, la "vision " des hommes du passé révolu et les processus cognitifs qui y président. Ainsi, le faux en préhistoire, dès lors qu'il se déclare et s’avoue comme tel, n’a pas toujours une valeur négative ${ }^{57}$.

55. De façon générale, «la recréation architecturale apparaît comme un levier pour accompagner la valorisation patrimoniale du site industriel requalifié », Gasnier, 2019, p. 334.

56. Malgat, Duval-Massaloux, Gauchon, 2012, p. 99-114.

57. Cohen, 1999, p. 31-40. 
Ces conclusions peuvent aisément être transposées dans le cas du projet mené à Rochefort. Comme avec la restitution des grottes préhistoriques, il ne s'agit pas de duper le public qui doit être capable de comprendre immédiatement que ce qu'il visite est une restitution, le faux ne cherchant pas à se faire passer pour du vrai. En revanche, le moulin, parce qu'il reprend l'essence même du principe conçu par Jean-Baptiste Hubert, doit devenir un outil d'intermédiation pour la compréhension des problématiques techniques locales et, plus globalement, du processus d'innovation. À défaut d'une authenticité, au sens de l'Unesco ${ }^{58}$, et d'une ancienneté qui mettra du temps à être acquise, la capacité didactique, voire sensorielle et émotionnelle, de cette restitution lui procurera une valeur patrimoniale indéniable dans la mesure où elle est susceptible d'une appropriation rapide par les visiteurs. À rebours d'une lecture trop stricte des Chartes d'Athènes et de Venise, les restitutions peuvent être considérées comme une chance pour la préservation du patrimoine " ancien ». L'attrait voire l'enthousiasme qu'elles suscitent, autant dans leur conception que dans leur exploitation, et le moteur qu'elles représentent pour la mise en tourisme d'un espace sont des gages pour relancer ou pérenniser la protection du patrimoine existant. Le projet de l'AMAR a l'ambition de jouer ce rôle dans la stratégie de requalification touristique et culturelle de l'arsenal de Rochefort.

Après trois années de réflexion et d'échanges, d'études préparatoires et de recherche de soutiens, l'année 2020 a représenté une étape majeure pour la reconnaissance du projet de restitution du moulin. En dépit des réserves de l'Architecte des bâtiments de France, le Conseil communautaire de la Communauté d'agglomération Rochefort Océan (CARO) a voté à l'unanimité une modification du PSMV qui autorise le principe de la construction de ce nouvel élément ${ }^{59}$. Cette décision ne vaut pas autorisation administrative pour l'engagement des travaux mais admet la possibilité de l'inclure au paysage de l'arsenal, sans remettre en cause le PSMV et la can-

\footnotetext{
58. Sur la question de «l'authenticité », fortement débattue actuellement : Boccardi, 2019, p. 4-18 et Mairesse, Peters, 2019 (http://network.icom.museum/fileadmin/user_upload/minisites/icofom/ images/Icofom-EssenceofConservation-FINAL.pdf)

59. Ainsi, la décision de la CARO indique que des " équipements, installations ou constructions illustrateurs de savoirs et innovations de l'Arsenal, pourraient être installés et mis en œuvre dans une démarche liée au développement durable et compatible avec les activités touristiques existantes et à venir. À ce titre, l'évocation d'un moulin à draguer la vase, inspiration du moulin d'Hubert, pourrait trouver place en bordure de Charente. "
} 
didature à l'obtention du label Grand site. Cette première étape a permis à l'AMAR de s'attacher les services d'un assistant de maîtrise d'ouvrage après le lancement d'un avis d'appel public à la concurrence et une épreuve en loge pour départager les candidats qui s'est déroulée le 7 février 2020. Si l'AMAR conserve la maîtrise de la réalisation du projet, cette entreprise mandataire assurera la conception et la réalisation du moulin, notamment en coordonnant l'ensemble des opérateurs qui interviendront sur le chantier, afin de proposer une architecture qui s'intègre à l'environnement et qui réponde aux objectifs de la restitution ${ }^{60}$. Ces deux étapes ne lèvent pas les incertitudes quant à la construction puisque la forme du moulin et du bateau racleur, les matériaux employés ainsi que le rassemblement du financement seront déterminants pour lever les derniers obstacles et obtenir les autorisations administratives. Néanmoins, au-delà de l'opportunité de redynamiser le site de l'arsenal en attirant un public nouveau, l'ensemble des parties prenantes ont intégré la capacité du moulin à devenir un outil de médiation en histoire des techniques et de l'innovation.

\section{Annexe}

Le Meunier et le Moulin à Vent, conte en vers, lu à la Société de Littérature, Sciences et Arts de Rochefort au cours de la séance du 22 mai 1810

Honneur! Honneur ! à l'homme de Génie qui pour l'État employant ses moyens

fait partager à ses Concitoyens

les heureux fruits de sa noble industrie

Vous connaissez tous ce Moulin à Vent

cette machine utile, ingénieuse,

dont un jeune homme et modeste et savant

fit dans ce port l'épreuve merveilleuse

là chaque jour, admirant ses effets

on peut aussi calculer ses bienfaits.

par le secours de la simple machine

en double sens la drague qui chemine

cure en tout temps le devant du bassin ;

60. À l'issue de l'épreuve en loge, c'est le groupement Asselin SAS qui a été sélectionné pour la conception du moulin et la gestion du futur chantier. Les détails sont disponibles sur le site de l'AMAR : http://moulin-arsenal.fr/wordpress/. 
et du limon que le fleuve y transporte, de jour et de nuit, débarrassant la porte dans le courant le reverse, où soudain l'eau qui s'enfuit le délaye et l'emporte. Là de vingt bras épargnant les sueurs, sur leur pivot quatre meules tournantes par le produit de leurs forces constantes servent encore à broyer les couleurs. D'un laminoir, là, deux rouleaux mobiles sous leurs efforts, retenant et pressant un plomb épais, de ses masses ductiles font une feuille en les amincissant. De la campagne on voyait ce prodige. A son sujet, chez un meunier voisin, S'élève un jour un assez long litige. Pour expliquer quel étoit ce moulin On dissertait, on ne devinait guères. De nous frauder aurait-on le dessein ? Et des meuniers envierait-on le pain? Je veux demain réunir mes confrères et nous irons ensemble à Rochefort voir ce Rival qu'on a mis dans le port. Deux jours après les meuniers se rendirent en cette ville; au chef du port ils dirent qu'ils arrivaient avec l'intention d'aller de suite y voir de compagnie le dit moulin, objet de leur envie. J'approuve fort la résolution répond ce chef, soit dit sans flatterie, il est bien fait pour l'admiration des gens de l'art experts dans la partie. Je vous accorde à tous permission de visiter cet œuvre de Génie. Tous les meuniers l'ayant examiné par le dehors, tourné puis retourné chacun d'abord argumente et divague sur l'ajouté qu'on y fit d'une drague 
partout ailleurs moyen inusité.

Ils sont instruits de son utilité

par le gardien, toujours sur le qui vive

pour en jaser, en tirant vanité

comme s'il eut ce moyen inventé

tel le bedeau d'un bon curé de Brive

croyait toujours lui-même avoir prôné

un beau sermon quand il l'avait sonné.

Mais direz vous notre conteur dérive, de la Charente il nous conduit à Brive.

J'ai pu risquer une comparaison,

le vieil Homère en a fait à foison,

c'est notre maitre, il faut bien qu'on le suive,

à mes meuniers je retourne et j'arrive,

ainsi qu'un autre en vient à ses moutons.

Ils sont contents de l'imaginative,

et de l'effet de cette drague active

le bon moyen, nous en profiterons

pour nos fossés, qu'ainsi nous curerons.

On entre... Peignez-vous leur surprise

tout est nouveau pour eux dans ce moulin

ils n'y voyaient rien qui fut à leur guise.

Des meules ! ...bon... mais où est donc le grain ?

Où sont les sacs? Nous les cherchons en vain.

Dans nos moulins, on moud et l'on tamise,

Dans celui-ci que fait-on donc enfin?

Oh qu'est ceci ? (nouveau sujet de crise

car rien n'échappe à leur prompt examen)

cette couleur rougeâtre jaune ou grise

que chaque meule et broie et pulvérise

est pour eux tous matière à bien du train.

Mais raisonner dès ors chacun se presse,

Ils péroraient, ils disputaient sans cesse,

c'était un bruit à rendre les gens sourds.

Quand le plus vieux demanda du silence

Pour un avis de très grande importance

Car c'est ainsi qu'il débutait toujours 
On fait le cercle, on lui donne audience,

On s'en rapporte à son expérience

Or mot à mot voici tout son Discours

"Sans décider ici chacun opine

"Écoutez donc Messieurs votre Doyen

"Ce faux moulin (et je m'y connais bien)

"est pour la montre une belle machine

"car tout moulin qui ne fait pas farine

”à mon avis certes n'est bon à rien.

"Si tels étaient les vôtres et le mien

" en peu de temps nous aurions la famine.

"Son mécanisme est drôle assurément,

"Je sais qu'ici bien des gens en sont ivres

"Concevez-vous un pareil engouement?

"Amis, Voici mon dernier argument

"À son auteur, qui fit avec ses livres

"ce beau chef-d'œuvre, on doit remerciement,

"car s'il servait à moudre orge et froment

"il nous aurait bientôt coupé les vivres.

"Mais il le fit, et fort heureusement

"pour notre emploi, pour pêcher en eau trouble.

"Or nos moulins nous valent plus du double

"par la mouture, et vous savez comment.

Bravo! Bravo! Dit l'assemblée entière,

nous sommes tous de votre sentiment,

puis les bons mots et l'ironie amère

"le beau moulin !...point n'y faut de meunière

"ni de mulets dit un autre plaisant

"car ces Messieurs ont pour eux la rivière

"et savent bien tirer partie du Vent.

Bref, décidé, même unanimement

Que ce moulin en aucune manière

N'est préférable aux nôtres. Cependant

les Gens de Ville en pensent autrement

mais des Meuniers ce n'est pas là l'affaire.

Que dites-vous, Messieurs, du Jugement

Ainsi porté par la gent farinière? 
Sur ce qu'on voit assez communément

A réfléchir moi j'y trouve matière.

De tant de gens qui sont d'avis contraires,

ce conte-ci nous apprend le secret.

Ce que l'un vante à quelqu'autre déplait.

Est-ce le goût qui fait que l'on diffère

d'opinion, et que l'on délibère?

Eh ! Non, vraiment, Messieurs, c'est l'intérêt,

C'est lui qui plaide et qui dicte l'arrêt.

Croizetière, Procès-verbaux des Travaux de la Société de Littérature, Sciences et Arts de Rochefort, 22 mai 1810, Bibliothèque de la Société de Géographie, Rochefort.

\section{Sources}

Blondel François, Cours d'architecture enseigné dans l'Académie royale d'architecture, chez Pierre Audoin \& François Clouzier, Paris, 1675, chapitre XIII, " Digressions sur les Bâtimens de Marine à Rochefort » et chapitre XIV, « De la Corderie de Rochefort \& de la manière de fonder sur grilles ".

Dupin Charles, "Rapport fait à l'Académie des sciences de l'Institut de France sur les nouvelles machines de M. Hubert, ingénieur de la Marine ", Annales Maritimes et Coloniales, Imprimerie nationale, Paris, 1818, p. 939-950.

Forfait Pierre-Alexandre, "Port ou havre ", Encyclopédie méthodique marine, tome III, chez Panckoucke, Paris, 1787, p. 189-211.

Romme Charles, Dictionnaire de la Marine françoise, chez P.L. Chauvet/Barrois l’aîné, La Rochelle/Paris, 1792.

\section{Bibliographie}

Abiven Marie-Morgane, "Humanités numériques et méthodes de conservation et de valorisation du patrimoine maritime : l'exemple des arsenaux de Brest et de Venise ", doctorat sous la direction de Garlatti Serge et LaubÉ Sylvain, université de Brest, 2019.

Acerra Martine, Arsenal et Patrimoine. L'Europe atlantique et Rochefort, Les Indes savantes, collection Rivages des Xantons, Paris, 2009.

Acerra Martine, "Rochefort : l'arsenal, l'eau et les vaisseaux ", dans Acerra Martine, Mérino José, Jean Meyer (dir.), Les Marines de guerre européennes, XVII ${ }^{e}$-XVIII siècles, Presses universitaires de Paris-Sorbonne, Paris, 1985, 1998, p. 63-73. 
ACERra Martine, Rochefort et la construction navale française, 1661-1815, Librairie de l'Inde, Paris, 1993.

Acerra Martine, "Les arsenaux français de marine à la fin du XVIII siècle ", dans Bonel Ulane (dir.), Fleurieu et la Marine de son temps, Economica, Paris, 1992, p. 179-189.

BARJOT Dominique, La grande entreprise française de travaux publics (1883-1974), Economica, Paris, 2006.

BoccArdi Giovanni, "Authenticity in the heritage context: a reflection beyond the nara document", The Historic Environment: Policy \& Practice, $\mathrm{n}^{\circ} 10-1$, 2019, p. 4-18.

Boito Camillo, Conserver ou restaurer : les dilemmes du patrimoine, Les éditions de l'imprimeur, Besançon, 2000.

Brisou Dominique, Accueil, introduction et développement de l'énergie vapeur dans la Marine militaire française au XIX siècle, Service historique de la Marine, Paris, 2001.

Cabantous Alain, "L'histoire maritime : objet de recherche ou leurre historiographique ", dans Villain-Gandossi Christiane et Rieth Éric (dir.), Pour une histoire du "fait maritime": sources et champs de recherche, CTHS, Paris, 2001, p. 33-43.

Charpy Jacques, Un ingénieur de la Marine au temps des Lumières. Les carnets de Pierre Toufaire (1777-1794), Presses universitaires de Rennes, Rennes, 2011.

Cohen Claudine, "Faux et authenticité en préhistoire ", Terrain, Anthropologie et Sciences humaines, $\mathrm{n}^{\circ} 33$, septembre 1999, p. 31-40.

Coтte Michel, "Les techniques numériques et l'histoire des techniques : le cas des maquettes virtuelles animées ", La Numérisation du patrimoine technique, Documents pour l'histoire des techniques, nouvelle série, $\mathrm{n}^{\circ} 18,2^{\mathrm{e}}$ semestre 2009, p. 7-21.

Dupont Maurice, Fardet Marc, L'Arsenal de Colbert : Rochefort, Centre international de la mer, La Rochelle, 1993.

EDgerton David, Quoi de neuf? Du rôle des techniques dans l'histoire globale, Seuil, Paris, 2013.

Gasnier Marina, «Réflexion épistémologique sur le patrimoine industriel : de la pluridisciplinarité à l'interdisciplinarité ", Revue d'histoire des sciences, $\mathrm{n}^{\circ} 2$, tome 72, 2019, p. 309-347.

Gasnier Marina, Le Patrimoine industriel au prisme de nouveaux défis : usages économiques et enjeux environnementaux, Presses universitaires de Franche-Comté, Besançon, 2018.

Gasnier Marina, Patrimoine industriel et technique. Perspectives et retour sur 30 ans de politiques publiques au service des territoires, Lieux Dits Éditions, Cahier du Patrimoine $\mathrm{n}^{\circ}$ 96, Paris, 2011. 
Gille Bertrand, "La notion de "système technique” (essai d'épistémologie technique) ", Culture technique, n 1,1979 , p. 8-18.

Jarrige François et VRIgnon Alexis (dir.), Face à la puissance. Une histoire des énergies alternatives à l'âge industriel, La Découverte, Paris, 2020.

Kerfridin Rémi et Cros Bernard, L'Arsenal de Toulon : quais, industrie et patrimoine, Extrême Éden Édition, Toulon, 2011.

Le Gall Céline, Giovanni Poleni (1683-1761) et l'essor de la technologie maritime au siècle des Lumières, Brepols, Turnhout, 2019.

Llinarès Sylviane et Hrodej Philippe, "La mer et la guerre à l'époque moderne », La Recherche internationale en histoire maritime : essai d'évaluation, Revue d'histoire maritime, PUPS, Paris, 2010, p. 317-339.

Mairesse François et F. Peters Renata, What is the Essence of Conservation? Materials for a Discussion, 25 congrès de l'ICOM-CC et de l'ICOFOM (Kyoto, 4 septembre 2019), Paris, ICOFOM, 2019.

Malgat Charlotte, Duval-Massaloux Mélanie et Gauchon Christophe, "Donner à voir un patrimoine invisible : de l'original à la copie. Le cas de l'espace de restitution de la grotte Chauvet-Pont-d'Arc. ", Cahiers de géographie, $\mathrm{n}^{\circ}$ 13, 2012, p. 99-114.

MÉmain René, La Marine de guerre sous Louis XIV : le matériel : Rochefort, arsenal modèle de Colbert, Hachette, Paris, 1937.

Montel Nathalie, "Le Chantier du canal de Suez (1859-1869). Techniques et techniciens de travaux publics au milieu du XIX ${ }^{\mathrm{e}}$ siècle ", thèse sous la direction d'André Guillerme, CNAM, 1995.

Morera Raphaël, L'Assèchement des marais en France au XVII siècle, Presses universitaires de Rennes, Rennes, 2011.

Morera Raphaël, "La politique hydraulique des rois de France au XVII siècle. Des constructions d'emblèmes ", Hypothèses, n 1, 2005, p. 49-60.

Ortholan Henri, Cros Bernard et GéHan Thierry, Les Ingénieurs de l'infrastructure militaire : cinq siècles d'histoire, Service historique de la Défense, Paris, 2012.

PARK Rang-Ri, "La société de construction des Batignolles, des origines à la Première Guerre Mondiale (1846-1914) : premiers résultats ", Histoire, économie et société, n' 19-3, 2000, p. 361-386.

Picon Antoine, L'Invention de l'ingénieur moderne. L'École des Ponts et Chaussées, 1747-1851, Presses de l'École nationale des ponts et chaussées, Paris, 1992.

Plouviez David, La Marine française et ses réseaux économiques au XVIIr siècle, Les Indes savantes, collection Rivages des Xantons, Paris, 2014.

Ponsonnet Bruno (dir.), L’Arsenal de Rochefort, Musée national de la Marine, Paris, 2008.

SCHAFfER Simon, "The charter'd Thames": naval architecture and experimental spaces in Georgian Britain ", dans Roberts Lissa, Schaffer Simon 
et Dear Peter (dir.), The Mindful Hand. Inquiry and Invention from the Late Renaissance to Early Industrialisation, Koninklijke Nederlandse Akademie van Wetenschappen, Amsterdam, 2007, p. 279-305.

Vacant Claude, Jean-Rodolphe Perronet (1708-1794) : "Premier ingénieur du Roi » et directeur de l'École des Ponts et Chaussées, Presses de l'École nationale des ponts et chaussées, Paris, 2006.

\section{Les auteurs}

Pierre Gras est à l'origine ingénieur en organisation et gestion des entreprises. Il occupe ensuite différentes fonctions dans des entreprises avant de s'engager dans l'Association Hermione-La Fayette, où il est membre du comité technique. Abordant alors les difficultés liées à l'envasement de la Charente, il propose de reproduire l'idée de Jean-Baptiste Hubert qui conduit à la création de l'Association du Moulin de l'Arsenal de Rochefort, dont il est le président depuis sa création. Contact : pierregras@outlook.com

David Plouviez est maître de conférences en histoire moderne à l'université de Nantes et membre du Centre de recherches en histoire internationale et Atlantique (CRHIA EA 1163). Ses travaux portent sur les modalités économique, sociale et technique de l'édification des flottes de guerre européennes du $x v I^{e}$ siècle au début de la première industrialisation. Ses recherches actuelles sorientent plus particulièrement sur les conditions du travail portuaire dans les ports de commerce et les arsenaux, notamment dans le contexte des chantiers navals.Contact : david.plouviez@univ-nantes.fr

Denis Roland est attaché de conservation du patrimoine. De 2003 à 2018, il est en charge des collections et de la médiation au musée national de la Marine et à l'École de médecine navale à Rochefort. À ce titre, il travaille sur l'histoire de l'arsenal maritime de Rochefort dans ses diverses dimensions : techniques, politiques, sociales, militaires ou imaginaires. Il est actuellement vice-président de l'Association du Moulin de l'Arsenal de Rochefort. Contact : roland.denis@ wanadoo.fr 